\title{
Multicentric Castleman’s Disease with Mycobacterium cosmeticum Infection
}

\author{
Anand AS*, Kuriakose VG, Govindan K, Nachimuthu K, John RM and Meloot SS
}

Department of Radiotherapy and Clinical Oncology, Government Medical College, Thiruvananthapuram, Kerala

"Corresponding author: Anand AS, Department of Radiotherapy and Clinical Oncology, Government Medical College, Thiruvananthapuram, Kerala, India, Tel: + 91-471-2528386; E-mail: anandrt2006@yahoo.com

Received date: Oct 31, 2016; Accepted date: Jan 31, 2017; Published date: Feb 7, 2016

Copyright: $\odot 2017$ Anand AS, et al. This is an open-access article distributed under the terms of the Creative Commons Attribution License, which permits unrestricted use, distribution, and reproduction in any medium, provided the original author and source are credited.

\begin{abstract}
Multicentric Castleman's Disease (MCD) is a rare and poorly understood disorder that straddles the intersections of hematology, oncology, rheumatology, and virology and can be fatal if improperly treated. The etiology of HHV8 negative disease is unknown and only isolated cases of associations with infectious agents have been reported in literature. However the co-occurrence of Mycobacterium cosmeticum with Castleman's disease has never been previously reported in literature. Hence we would like to report a case of Multicentric Castleman's disease with a coexisting M. cosmeticum infection.
\end{abstract}

Keywords: Ulcerative colitis; Imageology; Hypoalbuminemia; Follicles

\section{Case report}

58 year old gentleman, a painter by profession, who gives a history of ulcerative colitis for the past six years, was evaluated in medical gastroenterology department of our institution in October 2016, for symptoms of progressively increasing abdominal distension and loss of weight for the past eight months [1].On clinical examination he had right cervical Level II Lymph node enlargement $(2 \times 1.5 \mathrm{~cm})$ with moderate ascites and splenomegaly. All other systems were unremarkable on examination. At the time of initial presentation his laboratory investigations revealed hypoalbuminemia, with all other parameters within normal limits including ECHO scan.

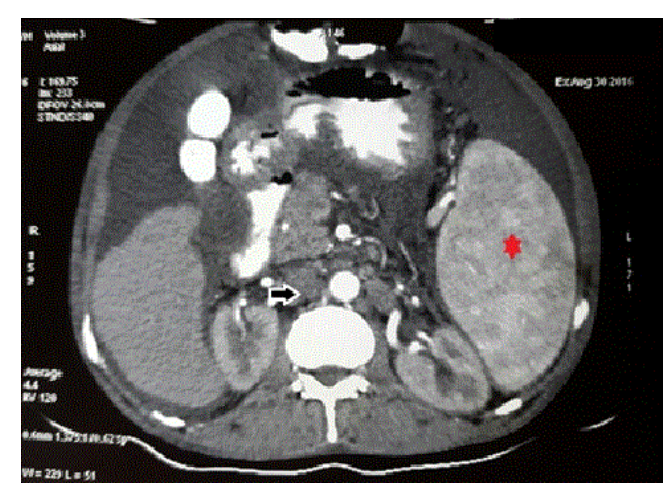

Figure 1: CECT Abdomen-paraaortic lymphadenopathy (black arrow) and splenomegaly (red star).

His physical examination findings were confirmed on imageology with Contrast Enhanced Computed Tomography (CECT) abdomen revealed features of hepatomegaly with cirrhotic changes, features of portal hypertension, ascites and moderate splenomegaly. Multiple enlarged upper and lower para aortic lymphadenopathy with the largest in the upper paraaortic region measuring $1.8 \times 1.2 \times 2.5 \mathrm{~cm}$ (Figure 1). The features of chronic liver disease seen in this patient was due to the Hepatitis $\mathrm{C}$ infection, confirmed by the detection of anti HCV antibody.

Viral markers were negative for HIV, HBsAg and HHV8.

Upper GI endoscopy displayed features of severe portal hypertensive gastropathy and antral erosions. Biopsy was negative for Helicobacter pylori infection. Colonoscopy examination revealed some areas of ulceration in the sigmoid colon with histopathology suggestive of ulcerative colitis.

Ascitic fluid cytology study was negative for malignant cells, with ascitic fluid culture revealing Acid Fast Bacilli (AFB) of Mycobacterium sp. Gene expert test to identify Mycobacterium Tuberculosis was negative. Special identification test (MALDI-TOF) was done which identified AFB as Mycobacterium cosmeticum (Figure 2).

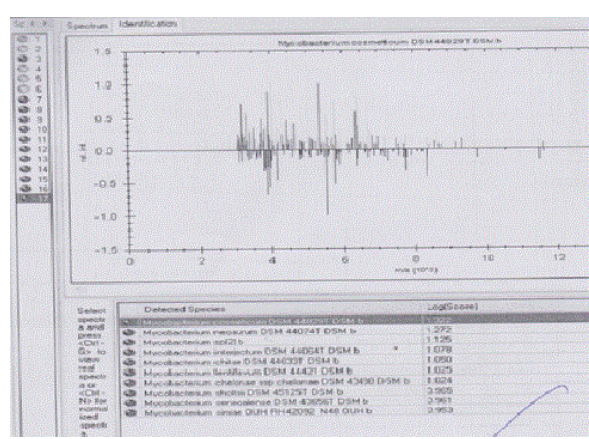

Figure 2: MALDI-TOF testing for Mycobacterium cosmeticum.

Biopsy from the cervical lymph node was suggestive of Castleman's disease (angiofollicular hyperplasia). On low power microscopy, an increase in the number of follicles in the cortex with the germinal 
Citation: Anand AS, Kuriakose VG, Govindan K, Nachimuthu K, John RM, et al. (2017) Multicentric Castleman's Disease with Mycobacterium cosmeticum Infection. Arch Surg Oncol 3: 120. doi:10.4172/2471-2671.1000120

Page 2 of 3

centre showing depletion of lymphocytes was noticed following hematoxylin and Eosin (H\&E) staining (Figure 3).

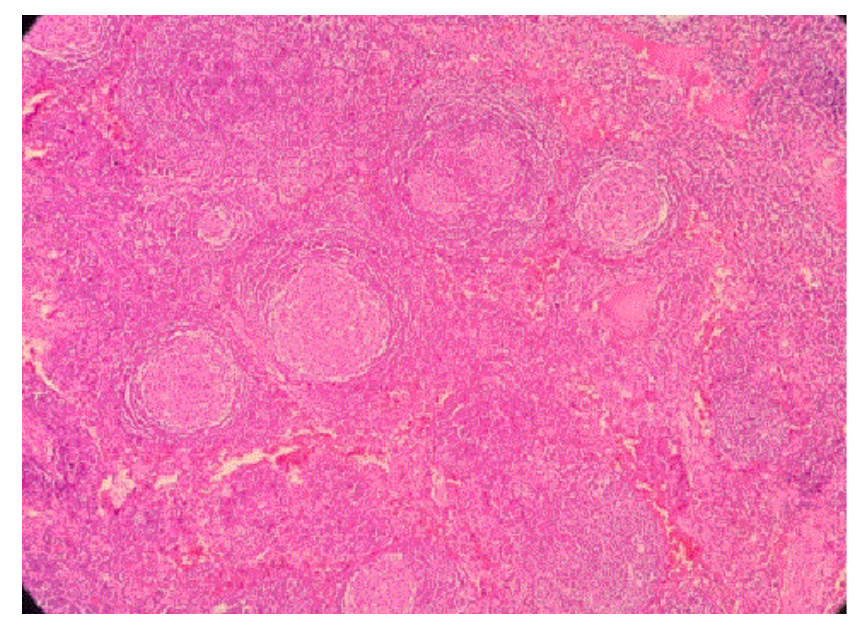

Figure 3: Low power microscopy (10X)-increase in number of follicles in the cortex with lymphocyte depletion in the germinal centre.

High power microscopy displayed the characteristic concentric layering of lymphocytes in the mantle zone (onion skin appearance) and germinal centre shows depleted lymphocytes with proliferated follicular dendritic cells with vesicular nucleus (Figure 4).

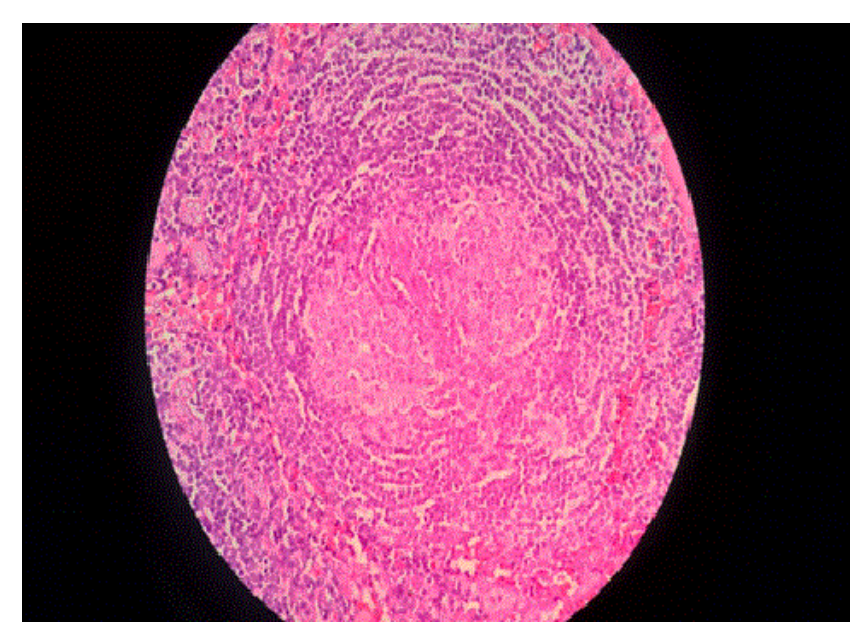

Figure 4: High power microscopy (40X)-characteristic onion skin appearance of concentrically arranged lymphocytes in the mantle zone.

Special reticulin staining showed vascular proliferation and transgressing of follicles by blood vessels (Figure 5).

The above histopathological findings strongly indicate to the diagnosis of Castleman's disease.

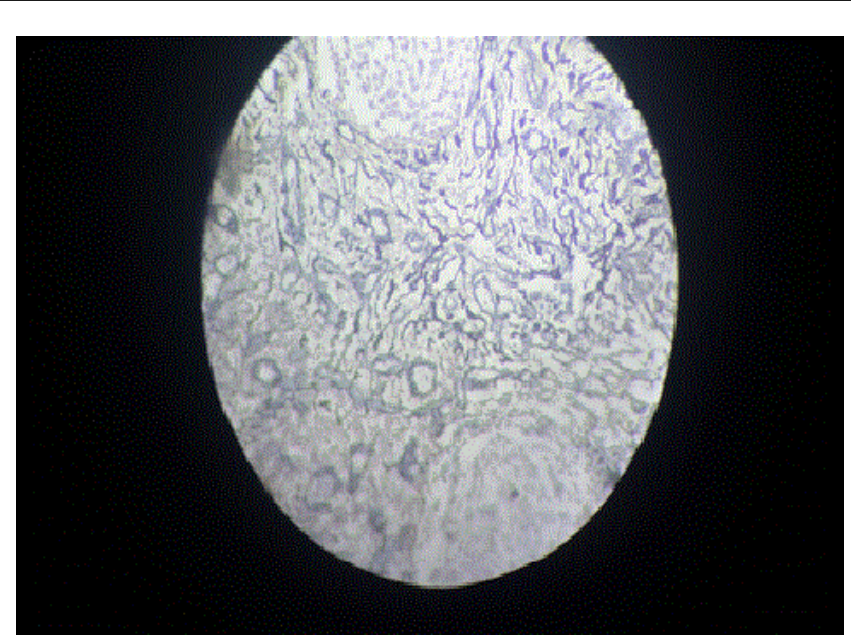

Figure 5: Reticulin staining under high power microscopy (40X)vascular proliferation and transgressing of follicles by blood vessels.

Considering the clinical, pathological, microbiological and radiologically evidences we arrived at the diagnosis of Multicentric Castleman disease with $M$. cosmeticum infection. Patient is initially started on Clarithromycin $500 \mathrm{mg}$ twice daily for two weeks and $\mathrm{T}$ Lamivudine to continue. He has completed 3 of the planned 6 cycles of CHOP (Cyclophosphamide, Adriamycin, Vincristine, Prednisolone) chemotherapy regimen, which is one of the accepted treatment schedules for MCD. He has shown good clinical and symptomatic response.

\section{Discussion}

Mycobacterium cosmeticum which comes under the classification of rapidly growing mycobacteria belong to the group of schotochromogens. It was first isolated in 2004 from a culture of a sink drain in a nail salon and from a granulomatous skin lesion in a female patient who underwent mesotherapy in Venezuela [2]. Cases of skin and soft tissue infection involving M. cosmeticum sp. and following mesotherapy were also reported [3].

Castleman disease is named after Benjamin Castleman, who first described the characteristic histopathological findings of angiofollicular lymph node hyperplasia in a localized lymph node region in 1954. Castleman disease is classified as unicentric (UCD) and multicentric (MCD). UCD is often asymptomatic and has a high cure rate with surgical excision of the enlarged lymph node. Multicentric Castleman's Disease (MCD) describes a heterogeneous group of disorders involving proliferation of morphologically benign lymphocytes due to excessive pro-inflammatory hypercytokinemia, most notably of interleukin-6.

Human Herpes Virus-8 (HHV-8) drives the hypercytokinemia in all HIV-positive patients and some HIV-negative patients. There is also a group of HIV-negative and HHV-8-negative patients with unknown etiology and pathophysiology referred to as idiopathic MCD (iMCD). MCD histopathological features can be divided into 4 variants: Hyaline-Vascular (HV), Plasma Cell (PC), mixed, and plasmablastic. 
The clinical spectrum ranges from waxing and waning lymphadenopathy with B-symptoms to more severe cases involving intense inflammation, hepatosplenomegaly, vascular leak syndrome with anasarca, pleural effusions, and ascites, organ failure, and even death $[4,5]$.

Etiological factors from the available limited numbers of case reports and studies include a somatic chromosomal abnormality in non-neoplastic cells, germ-line genetic mutation in an inflammatory gene, ectopic IL-6 release by malignant tumor cells, autoantibody antigenic stimulation, and infectious agents. Epstein-Barr Virus (EBV), HHV-6, hepatitis B virus, cytomegalovirus, toxoplasma, and Mycobacterium tuberculosis have each been reported to co-occur with iMCD, even though most iMCD cases do not report an associated infectious agent [6-12].

\section{Conclusion}

Idiopathic MCD is HHV 8 and HIV negative. Several cases have reported a co-occurrence of several other viral \& bacterial organisms like mycobacterium tuberculosis with Castleman's disease. This is the first case of MCD to be reported having co-occurrence of a rare infection, $M$. cosmeticum. This infection may be pathologic, coincidental, or secondary to iMCD-related immune dysfunction. But the awareness of such a co occurence, should raise an index of suspicion with respect to the presence of causal relationship, if further clustering of similar cases are reported in the future.

\section{References}

1. Fajgenbaum DC, Rhee FV, Nabel CS (2014) HHV-8-negative, idiopathic multicentric Castleman disease: novel insights into biology, pathogenesis, and therapy. Blood 123: 2924-2933.
2. Cooksey RC, de Waard JH, Yakrus MA, Rivera I, Chopite M, et al. (2004) Mycobacterium cosmeticum sp. nov., anovel rapidly growing species isolated from acosmetic infection and from a nail salon. Int J Syst Evol Microbiol 54: 2385-2391.

3. Beer K, Waibel J (2009) Disfiguring scarring follow-ing mesotherapyassociated Mycobacterium cosmeticum infection. J Drugs Dermatol 8: 391-393.

4. Castleman B, Towne VW (1954) Case records of the Massachusetts General Hospital; weekly Clinico-pathological exercises; founded by Richard C. Cabot. N Engl J Med 251: 396-400.

5. Dispenzieri A, Armitage JO, Loe MJ, Geyer SM, Allred J, et al. (2012)The clinical spectrum of Castleman's disease. Am J Hematol 87: 997-1002.

6. Casper C (2005) The etiology and management of Castleman disease at 50 years: translating pathophysiology to patient care. Br J Haematol 129: 3-17.

7. Barozzi P, Luppi M, Masini L, Marasca R, Savarino M, et al. (1996) Lymphotropic herpes virus (EBV, HHV-6, HHV-8) DNA sequences in HIV negative Castleman's disease. Clin Mol Pathol 49: M232-M235.

8. Bowne WB, Lewis JJ, Filippa DA, Niesvizky R, Brooks AD, et al. (1999) The management of unicentric and multicentric Castleman's disease: a report of 16 cases and a review of the literature. Cancer 85: 706-717.

9. Chen CH, Liu HC, Hung TT, Liu TP (2009) Possible roles of Epstein-Barr virus in Castleman disease. J Cardiothorac Surg 4: 31.

10. Jones EL, Crocker J, Gregory J, Guibarra M, Curran RC (1984) Angiofollicular lymph node hyperplasia (Castleman's disease): an immunohistochemical and enzyme-histochemical study of the hyalinevascular form of lesion. J Pathol 144: 131-147.

11. Murray PG, Deacon E, Young LS, Barletta JM, Mann RB, et al. (1995) Localization of Epstein-Barr virus in Castleman's disease by in situ hybridization and immunohistochemistry. Hematol Pathol 9: 17-26.

12. Yuan XG, Chen FF, Zhu YM, Hu W, Zhao XY, et al. (2012) High prevalence of hepatitis B virus infection in HIV-negative Castleman's disease. Ann Hematol 91: 857-861. 